\title{
Democratizing Endoscopic Submucosal Dissection: Single-Operator Fully Robotic Colorectal Endoscopic Submucosal Dissection in a Pig Model
}

\author{
Pietro Mascagni, ${ }^{1}$ Sun Gyo Lim, ${ }^{2,3}$ Claudio Fiorillo, ${ }^{2}$ Philippe Zanne, ${ }^{4}$ Florent Nageotte, ${ }^{4}$ \\ Lucile Zorn, ${ }^{4}$ Silvana Perretta, ${ }^{1,2}$ Michel de Mathelin, ${ }^{4}$ Jacques Marescaux, ${ }^{1,2}$ and \\ Bernard Dallemagne
}

\begin{abstract}
${ }^{1}$ Research Institute against Digestive Cancer (IRCAD), and ${ }^{2}$ IHU Strasbourg, Institute of Hybrid Image-Guided Surgery, Strasbourg, France; ${ }^{3}$ Department of Gastroenterology, Ajou University School of Medicine, Suwon, Korea; and ${ }^{4}$ ICube, (UMR UDS-CNRS 7357), University of Strasbourg, Strasbourg, France
\end{abstract}

This article has an accompanying continuing medical education activity, also eligible for MOC credit, on page e17. (https://www. gastrojournal.org/cme/home). Learning Objective: Upon completion of this CME activity, successful learners will be able to recognize factors making endoscopic submucosal dissection (ESD) technically demanding and describe the potential role of robotic-assisted intraluminal surgical triangulation in leveraging the skills needed to perform such procedures.

Endoscopic submucosal dissection (ESD) is an
increasingly popular endoscopic procedure enabling en bloc resection of superficial cancers with a low risk of lymph node metastasis. Colorectal ESD is indicated for large premalignant lesions and intramucosal carcinomas or carcinomas with slight submucosal invasion. ESD achieves higher en bloc and curative resection rates than piecemeal EMR. ${ }^{1}$ Nevertheless, ESD is technically challenging and carries a $4.9 \%$ perforations rate. ${ }^{2}$ Although ESD has a well-established role in the treatment algorithm of most superficial colorectal neoplasms in Japan and East Asia, in Western countries, it is performed proficiently in only few centers and it is still a matter of debate. Western endoscopists are struggling to master the technical proficiency of their Eastern counterparts, because a high case volume and a long learning curve is required to master the procedure, especially in the colon. ${ }^{3}$ The lack of robust retraction, good exposure, and instruments triangulation accounts for ESD-related technical difficulties. Several methods and devices have been developed to overcome these technical limitations, including robotic assistance. ${ }^{4}$ In our institution, we have developed a robotic flexible endoscopy platform that enables complex endoscopic and transluminal surgery. ${ }^{5-7}$ To our knowledge, this is the first fully robotic master-slave manipulator for flexible endoscopy controlled by a single operator.

Between January and May 2018, a nonrandomized comparative animal trial was performed to compare the safety and efficacy of robot-assisted versus conventional colonic ESD. Twenty robotic-assisted colonic ESD carried out by a laparoscopic surgeon with no previous robotic or conventional ESD experience (experimental group) were compared with 10 ESD performed by an expert Asian endoscopist ( $>1000$ ESD) using conventional endoscopic equipment and technique (control group). The study was approved by the local ethics committee (ICOMETH $\left.\mathrm{N}^{\circ} 38.2016 .01 .082\right)$ and by the French Ministry of Superior Education and Research (APAFIS \#6168-201607 $2209464427 \mathrm{~V} 4 ; 24^{\text {th }}$ of November 2016). Thirty pseudotumors were created in the colon of 10 large white pigs (40 kg), 15-35 cm from the anal verge. Operative time, achievement of curative resection (R0), and complications were recorded.

In the robotic group, all ESD were completed with a $100 \%$ en bloc resection rate. In the control group, 1 procedure $(10 \%)$ was aborted owing to failure of submucosal lifting. The mean specimen size was comparable $(15.73 \pm$ $8.08 \mathrm{~cm}^{2}$ vs $14.07 \pm 4.09 \mathrm{~cm}^{2} ; P=.34$ ). Robotic assistance significantly increased the safety profile $(5 \%$ vs $33.3 \%$ perforation rate; $P=.041$ ) and dissection speed (57.05 \pm $29.42 \mathrm{~mm}^{2} / \mathrm{min}$ vs $35.21 \pm 16.20 \mathrm{~mm}^{2} / \mathrm{min} ; P=.049$; Figure 1), while decreasing both total procedural (33.36 \pm 14.20 minutes vs $47.38 \pm 13.40$ minutes; $P=.011$ ) and dissection times $(29.42 \pm 14.29$ minutes vs $43.43 \pm 12.55$ minutes; $P=.01$ ).

Abbreviationused in this paper: ESD, endoscopic submucosal dissection.

Most current article

\author{
(C) 2019 by the AGA Institute \\ 0016-5085/\$36.00 \\ https://doi.org/10.1053/j.gastro.2018.12.046
}




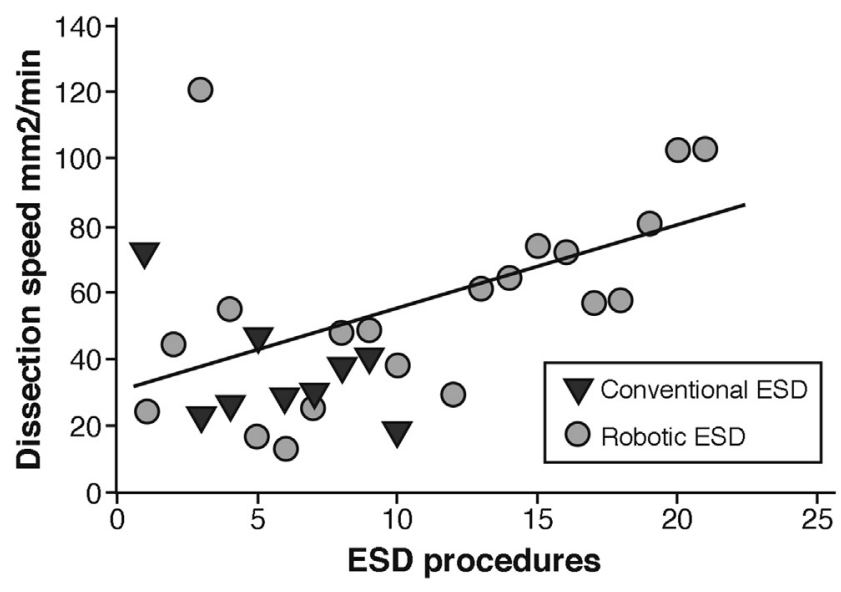

Figure 1. Dissection speeds in conventional (triangles) versus robotic (dots) endoscopic mucosal dissection (ESD). Robot assistance increases dissection speeds.

\section{Description of the Technology}

The Endoluminal Assistant for Surgical Endoscopy is a modular system composed of a master and a slave unit (Figure 2). An older version of the platform, referred to as STRAS, has been previously described ${ }^{5,6}$ and used for a single-arm study on colorectal ESD. ${ }^{7}$ The Endoluminal Assistant for Surgical Endoscopy was re-engineered to meet clinical and commercialization constraints. The slave unit consists of a mobile cart and a detachable flexible endoscope. The cart houses instruments modules and endoscope rotation/translation modules for robotic actuation. It is connected to the endoscope by means of a U-shaped arm. The 53.5-cm-long detachable endoscope has a maximum shaft diameter of $16 \mathrm{~mm}$ and houses two $4.3-\mathrm{mm}$ lateral working channels for telemanipulated flexible instruments and a third 3.2-mm central working channel for conventional endoscopic instruments. Robotic instruments have flexible tips controlled with antagonist tendons. The toolbox of instruments includes various graspers, a hook knife, an insulated tip knife, and an endoscopic needle holder. The distal jaw-like opening allows surgical triangulation spacing the exit of the instruments. The whole system has 10 degrees of freedom. The ergonomic design of the master unit allows a single operator sitting at the console to control both the endoscope and the instruments. Each joystick has 3 degrees of freedom to control robotic instruments, 4-way thumb switches to control endoscope/camera movements, and a trigger to control the opening and closing of working instruments. The operator has the endoscopic view on a frontal screen and a graphical user interface on a lateral screen mapping the position of the endoscope and instruments.

\section{Video Description}

Video 1 compares robotic-assisted versus conventional colorectal ESD, illustrating the key advantages of the robotic platform, including its stable view and intraluminal surgical triangulation. Thanks to robotic assistance, stable and robust traction and countertraction allow for the precise and effective dissection of the submucosal plane. The robotic enabled free rotation of the endoscope around its axis and provides a more stable view and an easier access to the target, irrespective of the lesion's location compared with standard ESD.

\section{Take-Home Message}

- ESD has created a bridge between endoscopy and surgery, providing access to "surgical" and "oncologic" values, such as $\mathrm{R} 0$ resection and complete remission of cancer.

- To our knowledge, this is the first fully robotic, single operator, master-slave telemanipulator for flexible endoscopy.

- This prospective comparative study showed that roboticassisted ESD is safer and faster than conventional ESD even when performed by a novice.

- The present work supports the evidence that flexible robotic technologies can enhance the performance of complex dissection within the gastrointestinal lumen applying surgical strategies and thinking.

- The Endoluminal Assistant for Surgical Endoscopy brings surgical proficiency within the lumen of the GI tract democratizing access to advanced organ sparring

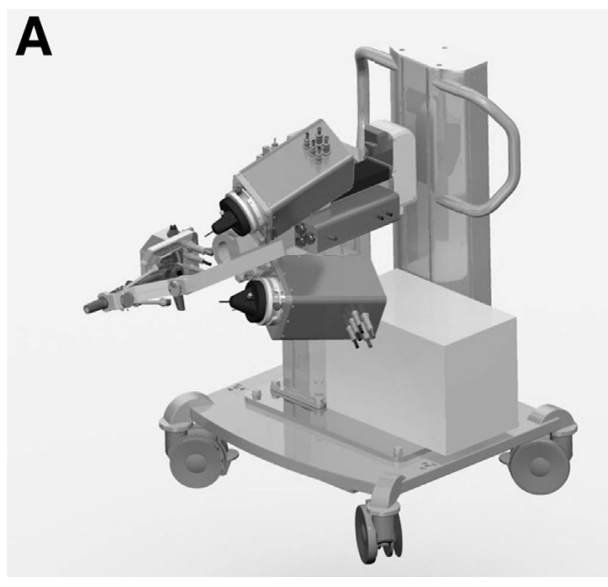

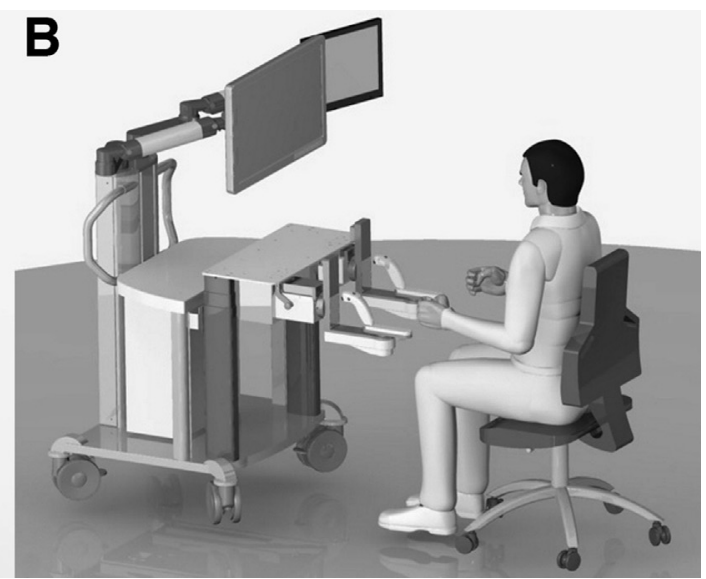

Figure 2. Virtual rendering of Endoluminal Assistant for Surgical Endoscopy. A, Slave unit: mobile cart with robotic modules. $B$, Master unit. 
GASTROENTEROLOGY IN MOTION

endoscopic procedures such as ESD for physicians and patients.

Supplementary Material

Note: To access the supplementary material accompanying this article, visit the online version of Gastroenterology at www.gastrojournal.org, and at https://doi.org/10.1053/ j.gastro.2018.12.046.

References

1. Watanabe T, Miro K, Ajioka Y, et al. Japanese Society for Cancer of the Colon and Rectum (JSCCR) guidelines 2016 for the treatment of colorectal cancer. Int J Chin Oncol 2018;23:1-34.

2. Arezzo A, Passera R, Marchese N, et al. Systematic review and meta-analysis of endoscopic submucosal dissection vs endoscopic mucosal resection for colorectal lesions. United European Gastroenterol J 2016;4:18-29.

3. Daoud DC, Ster N, Durand M, et al. Comparing outcomes for endoscopic submucosal dissection between Eastern and Western countries: A systematic review and metaanalysis. World J Gastroenterol 2018;24:2518-2536.

4. Young BPM, Chin PW. Application of robotics in gastrointestinal endoscopy: a review. World J Gastroenteral 2016;22:1811-1825.
5. De Bono A, et al. Introducing STRAS: a new flexible robotic system for minimally invasive surgery. IEEE International Conference on Robotics and Automation 2013:1213-1220.

6. Zorn L, Nageotte F, Kane P, et al. A novel telemanipulated robotic assistant for surgical endoscopy: preclinical application to ESD. IEEE Transactions on Biomedical Engineering 2018;65:797-808.

7. Légner A, Diana M, Halvax P, et al. Endoluminal surgical triangulation 2.0: A new flexible surgical robot. Proliminary preclinical results with colonic submucosal dissection. Int J Med Robot 2017;13(3).

Reprint requests

Address requests for reprints to: Bernard Dallemagne, MD, 1 place de l'Hôpital, 67091, Strasbourg, France. e-mail: bernard.dallemagne@ircad.fr.

Acknowledgments

lana Shutrova, Guy Temporal, and Christopher Burel offered their assistance for professional English proofreading.

Conflicts of interest

The authors disclose no conflicts.

Funding

Supported by a FUI project (ISIS) and by SATT-Conectus Alsace (EASE project). Parts of this work were supported by French state funds managed by the ANR within the Investissements d'Avenir program under the ANR-11LABX-0004 (Labex CAMI) and ANR-10-EQPX-44 (Robotex Equipment of Excellence).

1571 


\section{Supplementary Material}

\section{Study Design}

This was a prospective nonrandomized comparative preclinical study in an acute animal model performed in between January and May 2018 at IRCAD (Strasbourg, France). Robot-assisted colonic endoscopic mucosal dissections (ESD) performed by a laparoscopic surgeon without any prior experience with robotic and conventional ESD (B.D.) (Endoscopic Assistant for Surgical Endoscopy [EASE]-assisted group) were compared with colonic ESD performed with conventional endoscopic equipment by an endoscopist with a clinical expertise of $>1000$ ESD (S.G.L.) (conventional equipment group). The study was designed with a 2 (experimental arm) to 1 (control arm) procedure ratio to minimize biases derived from a higher likelihood of a learning effect in the EASE-assisted group. A total of 30 pseudotumors were delineated on the posterior, lateral, and upper walls of the colon at a distance from the anal verge ranging between 15 and $35 \mathrm{~cm}$. For logistic reasons, the series of robotic ESD was performed before the conventional series.

\section{Animals}

Ten large white pigs (Sus scrofa domesticus, ssp large white) were included in the study. All animals were managed according to the French laws for animal use and care and according to the directives of the European Community Council (2010/63/EU). The study was approved by the local ethics committee (ICOMETH $\mathrm{N}^{\circ} 38.2016 .01 .082$ ) and by the French Ministry of Superior Education and Research(APAFIS \#6168-2016072209464427v4; November 24, 2016). Animals were fasted for 24 hours before the procedure with free unlimited access to water. Tiletamine/zolazepam $(7.5 \mathrm{mg} / \mathrm{kg}$; Zoletil 100, Virbac, France) and azaperone (2 mm/kg; Stresnil, Janssen-Cilag, Belgium) were administered intramuscularly as premedication 10 minutes before the procedure. Induction of anesthesia was achieved using intravenous propofol ( $3 \mathrm{mg} / \mathrm{kg}$ ) combined with rocuronium $(0.8 \mathrm{mg} / \mathrm{kg})$. Anesthesia was maintained with $2 \%$ isoflurane after endotracheal intubation of the animals in the supine position. Rectal cleansing was performed until the effluent turned clear. All pigs were humanely killed immediately after the procedures with an intravenous injection of a lethal dose of potassium chloride.

\section{Procedures}

The main procedural ESD steps were the following: (0) scope manual insertion and navigation (through a GelPOINT Path Transanal Access Platform [Applied Medical, Rancho Santa Margarita, CA] for robotic-assisted ESD), (1) marking of the desired resection margins around fake tumors, (2) mucosal lifting via submucosal injection (23-G needle, Interject Contrast, Boston Scientific, MA) of saline $(\mathrm{NaCl}$ $0.9 \%$ ) solution with methylene blue, (3) precut of the mucosa, (4) submucosal dissection, (5) inspection of the colonic resection bed, and (6) extraction of specimens that were stretched, pin fixed, and examined.

\section{Experimental Outcomes}

The primary endpoints included intraprocedural complications rate (safety) and the complete en bloc dissection rate (efficacy). Secondary endpoints included total procedural time, dissection time, specimen size, and dissection speed (Supplementary Figure 1).

Colonic wall perforations and bleedings requiring clip applications were considered as complications. Complete en bloc resection was defined as the resection of a specimen containing the whole marked area in one piece.

Total procedural time was the time from the first mucosal marking to the complete removal of the fake tumor, and dissection time was calculated from the start of submucosal dissection. Dissection speed was defined as the ratio between specimen size and dissection time. Specimen size was measured on fresh specimens stretched and pin fixed on a corkboard.

All procedures were recorded. Data were collected during the procedures and reviewed on recordings.

\section{Statistical Analysis}

Continuous variables were reported as mean \pm standard deviation and categorical variables in numbers and percentage points unless otherwise specified. Ordinal qualitative and quantitative variables were compared with Wilcoxon or Kruskal Wallis sum of ranks test. Paired comparison of qualitative variables was performed with a Fisher's exact test or $\chi^{2}$ tests. A $P$ value of $<.05$ was required to conclude statistical significance. SPSS software, version 25.0 (IBM-SPSS, Chicago, IL), was used for the analysis. 


\section{GASTROENTEROLOGY IN MOTION}

\begin{tabular}{|c|c|c|c|c|c|c|c|c|c|c|}
\hline Animal & Sex & $\begin{array}{l}\text { Weight } \\
(\mathrm{kg})\end{array}$ & $\begin{array}{c}\text { ESD } \\
\text { location }\end{array}$ & $\begin{array}{c}\text { Distance } \\
\text { (cm) }\end{array}$ & $\begin{array}{c}\text { Complete } \\
\text { en bloc }\end{array}$ & Complications & $\begin{array}{c}\text { Procedure } \\
\text { time }\end{array}$ & $\begin{array}{c}\text { Dissection } \\
\text { time }\end{array}$ & $\begin{array}{l}\text { Specimen } \\
\text { size }(\mathrm{cm} 2)\end{array}$ & $\begin{array}{l}\text { Dissection } \\
\text { speed } \\
\text { (mm2/min }\end{array}$ \\
\hline \multicolumn{11}{|c|}{ Robot-assisted } \\
\hline \multirow{3}{*}{1} & \multirow{3}{*}{ female } & \multirow{3}{*}{30} & bottom & 30 & yes & perforation & $00: 27: 39$ & $00: 20: 39$ & 4.9 & 24,50 \\
\hline & & & \begin{tabular}{|l|} 
bottom \\
\end{tabular} & 25 & yes & none & $00: 12: 51$ & $00: 07: 27$ & 3.14 & 44,86 \\
\hline & & & bottom & 20 & yes & none & $00: 13: 20$ & $00: 08: 40$ & 9.7 & 121,25 \\
\hline \multirow{3}{*}{2} & \multirow{3}{*}{ male } & \multirow{3}{*}{35} & top & 25 & yes & none & $00: 33: 04$ & $00: 23: 22$ & 12.56 & 54,61 \\
\hline & & & lateral & 30 & yes & none & $00: 24: 59$ & $00: 18: 39$ & 3.14 & 17,44 \\
\hline & & & \begin{tabular}{|l|} 
bottom \\
\end{tabular} & 20 & yes & none & $00: 15: 14$ & $00: 13: 23$ & 1.76 & 13,54 \\
\hline \multirow{4}{*}{3} & \multirow{4}{*}{ female } & \multirow{4}{*}{30} & \begin{tabular}{|l|} 
bottom \\
\end{tabular} & 35 & yes & none & $00: 51: 12$ & $00: 47: 52$ & 12 & 25,53 \\
\hline & & & bottom & 30 & yes & none & $00: 44: 47$ & $00: 40: 17$ & 19.25 & 48,13 \\
\hline & & & top & 20 & yes & none & $00: 45: 05$ & $00: 41: 03$ & 20 & 48.78 \\
\hline & & & bottom & 15 & yes & none & $00: 38: 14$ & $00: 35: 34$ & 13.5 & 38,57 \\
\hline \multirow{3}{*}{4} & \multirow{3}{*}{ male } & \multirow{3}{*}{50} & \begin{tabular}{|l|} 
Lateral \\
\end{tabular} & 35 & yes & none & $01: 16: 23$ & $01: 11: 23$ & 20.8 & 29,30 \\
\hline & & & bottom & 25 & yes & none & $00: 41: 07$ & $00: 39: 11$ & 24 & 61.54 \\
\hline & & & bottom & 30 & yes & none & $00: 34: 07$ & $00: 31: 07$ & 20 & 64,52 \\
\hline \multirow{3}{*}{5} & \multirow{3}{*}{ female } & \multirow{3}{*}{50} & bottom & 30 & yes & none & $00: 30: 22$ & $00: 27: 32$ & 20 & 74,07 \\
\hline & & & bottom & 25 & yes & none & $00: 27: 09$ & $00: 24: 55$ & 18 & 72,00 \\
\hline & & & top & 25 & yes & none & \begin{tabular}{|l|}
$00: 31: 34$ \\
\end{tabular} & $00: 28: 34$ & 16 & 57,14 \\
\hline \multirow{3}{*}{6} & \multirow{3}{*}{ male } & \multirow{3}{*}{45} & \begin{tabular}{|l|} 
bottom \\
\end{tabular} & 25 & yes & none & $00: 33: 35$ & $00: 31: 10$ & 18 & 58,06 \\
\hline & & & \begin{tabular}{|l|} 
lateral \\
\end{tabular} & 30 & yes & none & $00: 33: 04$ & $00: 30: 34$ & 24.3 & 81.00 \\
\hline & & & \begin{tabular}{|l|} 
lateral \\
\end{tabular} & 35 & yes & none & $00: 24: 25$ & $00: 21: 25$ & 21.6 & 102,86 \\
\hline 7 & male & 60 & top & 25 & yes & none & $00: 33: 46$ & $00: 31: 11$ & 32 & 103,23 \\
\hline \multicolumn{11}{|c|}{ Conventional endoscopic equipment } \\
\hline 7 & male & 60 & bottom & 25 & yes & none & $00: 25: 42$ & $00: 22: 42$ & 16.4 & 71,30 \\
\hline \multirow{5}{*}{8} & \multirow{5}{*}{ female } & \multirow{5}{*}{30} & Lateral & 35 & no & $N / A^{*}$ & $01: 37: 51$ & $01: 26: 07$ & $\mathrm{~N} / \mathrm{A}^{*}$ & $N / A^{*}$ \\
\hline & & & bottom & 20 & yes & perforation & $00: 35: 40$ & $00: 32: 28$ & 7 & 21,87 \\
\hline & & & bottom & 15 & yes & none & $01: 08: 52$ & $01: 05: 52$ & 16.5 & 25,38 \\
\hline & & & bottom & 25 & yes & none & $00: 37: 38$ & $00: 36: 08$ & 16.5 & 45,83 \\
\hline & & & top & 25 & yes & perforation & $00: 52: 38$ & $00: 48: 32$ & 13.5 & 28,13 \\
\hline \multirow{2}{*}{9} & \multirow{2}{*}{ female } & \multirow{2}{*}{40} & bottom & 25 & yes & none & $00: 42: 14$ & $00-38: 46$ & 11.25 & 29.60 \\
\hline & & & bottom & 15 & yes & none & $00: 47: 41$ & $00: 43: 32$ & 15,9 & 36,98 \\
\hline \multirow{2}{*}{10} & 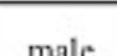 & 45 & \begin{tabular}{|l|} 
bottom \\
\end{tabular} & 25 & yes & perforation & \begin{tabular}{|l|}
$00: 58: 15$ \\
\end{tabular} & $00: 50: 31$ & 20 & 40,00 \\
\hline & & 45 & bottom & 35 & yes & none & $01: 000:(00)$ & $00: 54: 55$ & 9.62 & 17.81 \\
\hline
\end{tabular}

Supplementary Figure 1. Pig baseline presentation and ESD results. *Data not available because the procedure was aborted owing to failure of submucosal lifting. 